\title{
A study on impulse response model of reflected path for $\mathrm{THz}$ band
}

\author{
Kazuhiro Tsujimura*, Kenta Umebayashi*, Joonas Kokkoniemi ${ }^{\dagger}$, and Janne Lehtomäki ${ }^{\dagger}$ \\ * Tokyo University of Agriculture and Technology, Koganeishi, Tokyo \\ E-mail: kaz-260@st.go.tuat.ac.jp \\ $\dagger$ Centre for Wireless Communications (CWC), Oulu, Finland
}

\begin{abstract}
Impulse response based channel model is vital for wireless communication analysis and modeling. This paper considers the impulse response of the terahertz band ( $\mathrm{THz}$ band: 0.1-10 THz) for reflected path in case of short range $(1-100 \mathrm{~cm})$ wireless communication. In indoor application, it is necessary to consider multipath channel. In analysis of reflected path, rough surface of reflector is considered with Rayleigh roughness factor. The validity of the model is investigated with experimental $\mathbf{T H z}$ band measurements (up to $2 \mathrm{THz}$ ).
\end{abstract}

\section{INTRODUCTION}

The data rate requirements for the wireless communication increase exponentially. For instance, wireless local area networks will provide $10 \mathrm{Gbps}$ in near future [1], [2]. For ultra-high-speed communication, one of the candidates is the terahertz band (THz band: 0.1-10.0 THz) communications system. Lower frequency bands, such as ultra high frequency band $(300 \mathrm{MHz}-3 \mathrm{GHz})$, are utilized by many conventional wireless communication systems. The below $3 \mathrm{GHz}$ frequency bands have seen large numbers of channel models in the past [3]. In contrast to the UHF band, the effect of molecular absorption needs to be considered in the $\mathrm{THz}$ band [4].

Molecular absorption and its impact on transmittance of the channel were studied in [4]. Transmittance gives information about the fraction of the radiation capable of propagating through the channel and is modeled with the Beer-Lambert law. Thus, the Beer-Lambert law directly gives the molecular absorption loss in the channel [4]. Molecular absorption depends on the composition of a medium, relative humidity $(\mathrm{RH})$, pressure, and temperature, and it causes frequencyselective fading for wideband signals.

To develop wireless communication techniques for the $\mathrm{THz}$ band, the impulse response is required. One reasonable approach to obtain impulse response is to use transmittance with only amplitude information and no phase information. In [5], [6], [7], a linear phase was added to the transmittance and the impulse response was obtained by inverse Fourier transform (IFT). However, the impulse response based on a linear phase, or with no phase shift at all, does not satisfy causality. In order to satisfy causality, we added minimum phase shift to the transmittance and we obtained impulse response with causality from IFT [8]. In [8], we focused on the full terahertz band (0.1-10.0 THz) and consider communication distances suitable for short-range wireless communication (less than 1 meter) in line of sight (LoS) path and multipath scenarios. For both of

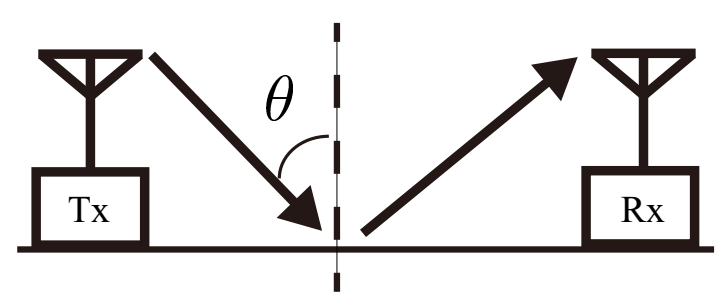

Fig. 1. Reflected path model from rough surface.

scenarios, we calculated frequency response and time response based on analytical approach. This paper provides frequency response and time response based on measurement result.

In an actual environment, we have to consider non line of sight (NLoS) path. Previous studies [9], [10] provided that reflected path is dominant effect in NLoS propagation. A reflected path model was derived with the Gaussian distribution based on rough surfaces [7]. However, they used linear phase shift to obtain the phase component. Therefore, the impulse response in [7] violates causality. In this paper we apply Kramers-Kronig relation to the reflected path model to satisfy with causality. We show the validity of our reflected path model by comparing with measurement data.

The rest of this paper is as follows. Section II describes the channel model of reflected path in $\mathrm{THz}$ band and in section III we derived phase component from amplitude component with causality. Section IV shows the validity of our proposed method for a reflected path. Section V concludes this paper.

\section{Channel MOdel}

We consider a reflected path from material with small conductivity (e.g. wood, glass) between transmitter and receiver as shown in Fig.1. The surface of the reflector is rough with respect to the wavelengths at the $\mathrm{THz}$ band. Reflected paths are derived from Kirchhoff's theory [2], [7]. Antenna characteristics are not considered in this model. We can describe frequency response $H_{\text {refl }}(f)$ as follows.

$$
H_{\mathrm{refl}}(f, z)=\left|H_{\mathrm{pl}}(f, z)\right| \cdot|R(f)| \cdot \exp (j \phi(f)),
$$

where $R(f)$ and $\phi(f)$ are the reflection coefficient and phase component in reflected path, respectively. $H_{\mathrm{pl}}(f, z)$ is the frequency response of path loss corresponding to the molecular absorption and spreading loss. Derivations of phase component 
by the conventional approach and our approach are described in section III.

The received signal $y(t)$ at time $t$ can be obtained by the convolution between the transmitted signal $x(t)$ and the impulse response, $h(\tau)$, as

$$
y(t)=\int_{-\infty}^{\infty} h(\tau) x(t-\tau) d \tau
$$

where $h(\tau, z)$ is the impulse response of the reflected path.

\section{A. Channel model of path loss in frequency domain}

The propagation distance of the reflected path is set to $z \mathrm{~cm}$. By considering the spreading loss and the molecular absorption loss, the power spectrum of path loss can be calculated as [4]

$$
\left|H_{\mathrm{pl}}(f, z)\right|^{2}=\left[A_{\text {abs }}(f, z) \times A_{\text {spread }}(z)\right]^{-1},
$$

where $A_{\text {spread }}(z)$ is the spreading loss and $A_{a b s}(f, z)$ is the molecular absorption loss. The spreading loss in the LoS path for an ideal isotropic transmitter is $A_{\text {spread }}(z)=4 \pi z^{2}$. $A_{a b s}(f, z)$ is given by [11]

$$
A_{a b s}(f, z)=\exp \left(\sum_{s} k_{a b s}^{s}(f) z\right),
$$

where $k_{a b s}^{s}$ is the absorption coefficient by the source of the absorption $s$. We utilize the am model [11], with the famous HITRAN catalog [12], to calculate the absorption coefficient.

\section{B. The reflection coefficient in frequency domain}

According to Kirchhoff's theory, the reflection coefficient $R(f)$ for a rough surface is given by

$$
R(f)=\gamma_{\mathrm{TE}}(f) \cdot \rho(f),
$$

where $\gamma_{\mathrm{TE}}(f)$ is the smooth surface reflection coefficient from the Fresnel equation for the transverse electric (TE) part of the electromagnetic (EM) wave and $\rho(f)$ is the Rayleigh roughness factor. Without loss of generality, this paper considers the TE part of the EM wave; the transverse magnetic (TM) part can be obtained by a similar approach. The smooth surface reflection coefficient is described by

$$
\gamma_{\mathrm{TE}}(f)=\frac{n_{i} \cos \theta-n_{t} \sqrt{1-\left(\frac{n_{i}}{n_{t}} \sin \theta\right)^{2}}}{n_{i} \cos \theta+n_{t} \sqrt{1-\left(\frac{n_{i}}{n_{t}} \sin \theta\right)^{2}}},
$$

where $n_{i}, n_{t}$ and $\theta$ are the refractive index of the air, the reflector and incident angle of the reflected path, respectively [13]. The refractive index of the reflector $n_{t}$ is frequency dependent. In this paper, $n_{i}=1$.

The rough surface effect is characterized by the Rayleigh roughness factor $\rho(f)$ as

$$
\rho(f)=\exp \left(-\frac{8 \pi \cdot f^{2} \cdot \sigma^{2} \cdot \cos ^{2} \theta}{c^{2}}\right) .
$$

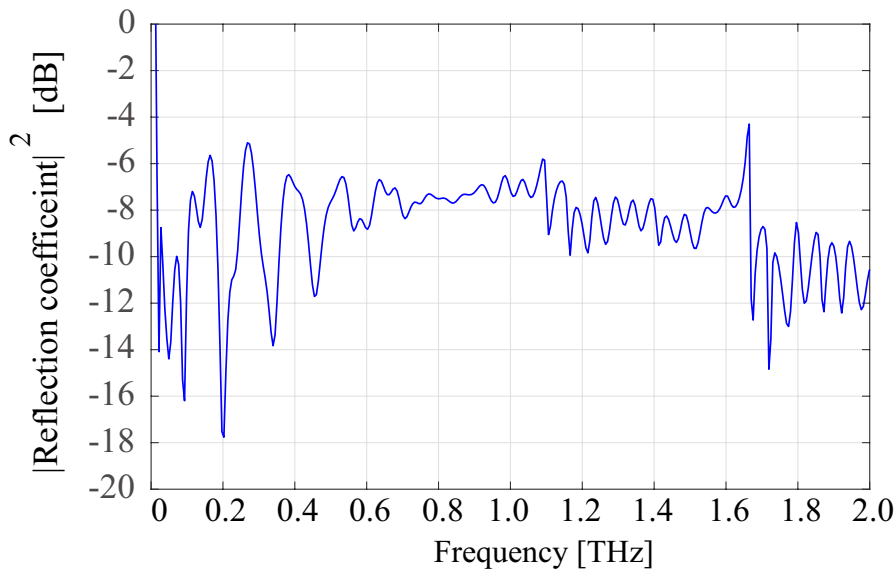

Fig. 2. Power spectrum of reflection coefficient from wood. Incident angle $\theta=60 \mathrm{deg}$.

We assume that the height of the rough surface has a Gaussian distribution with standard deviation $\sigma$. This assumption is valid for many indoor building materials [14].

In this paper, we calculated $R(f)$ based on $\mathrm{THz}$ time domain spectroscopy measurement data for which the measurement setup is described in section IV. $|R(f)|$ is calculated as bellow [9].

$$
|R(f)|^{2}=\frac{P_{\text {refl }}(f, z)}{P_{\text {samp }}(f, z)},
$$

$P_{\text {refl }}(f, z)$ and $P_{\text {samp }}(f, z)$ are the received power spectrum of reflected path and LoS path, respectively. For instance, Fig. 2 shows the power spectrum of reflection coefficient for smooth wood board coming from measurement data with incident angle $\theta=60 \mathrm{deg}$. Parameters used in this figure are distance $z=20 \mathrm{~cm}$, pressure $p=1007.9 \mathrm{hPa}, \mathrm{RH}=22.3 \%$, and temperature $T=295 \mathrm{~K}$.

\section{PHASE COMPONENT}

Two criterion to obtain the phase component in the frequency response are shown in this section. The first criterion is linear phase and the second criterion is minimum phase. In the linear phase, the phase component is determined by the time shift and the frequency. On the other hand, in minimum phase, we employ Kramers-Kronig relation to determine the phase component.

\section{A. Linear phase}

In the linear phase criterion, the phase component is set by $\phi_{\mathrm{L}}(f, z)=-2 \pi f z / c$. In this case, the frequency response $H_{\mathrm{L}}(f)$ is given by

$$
H_{\mathrm{L}, \mathrm{refl}}(f)=\left|H_{\mathrm{refl}}(f, z)\right| \cdot \exp \left(j \phi_{\mathrm{L}}(f, z)\right),
$$

where $\left|H_{\mathrm{L}, \mathrm{ref}}(f)\right|$ is the square root of the power spectrum of the reflected path found using (3) and (8). The impulse response $h_{\mathrm{L}, \mathrm{refl}}(\tau)$ is obtained by

$$
h_{\mathrm{L}, \mathrm{refl}}(\tau)=\mathcal{F}^{-1}\left[H_{\mathrm{L}, \mathrm{refl}}(f)\right],
$$




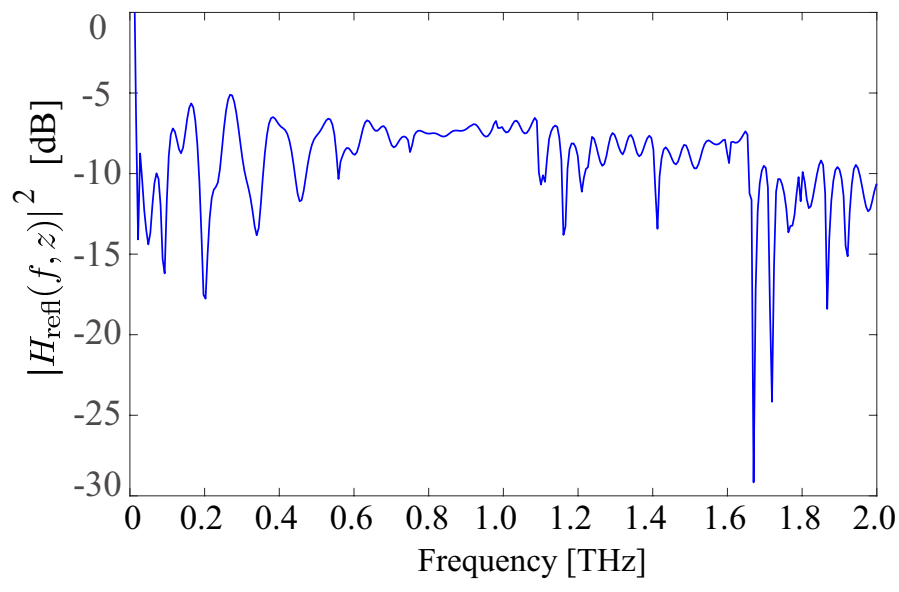

Fig. 3. Power spectrum of reflected path from wood. Incident angle $\theta=60$ $\mathrm{deg}$, distance $z=20 \mathrm{~cm}$, pressure $p=1007.9 \mathrm{hPa}, \mathrm{RH}=22.3 \%$, and temperature $T=295 \mathrm{~K}$.

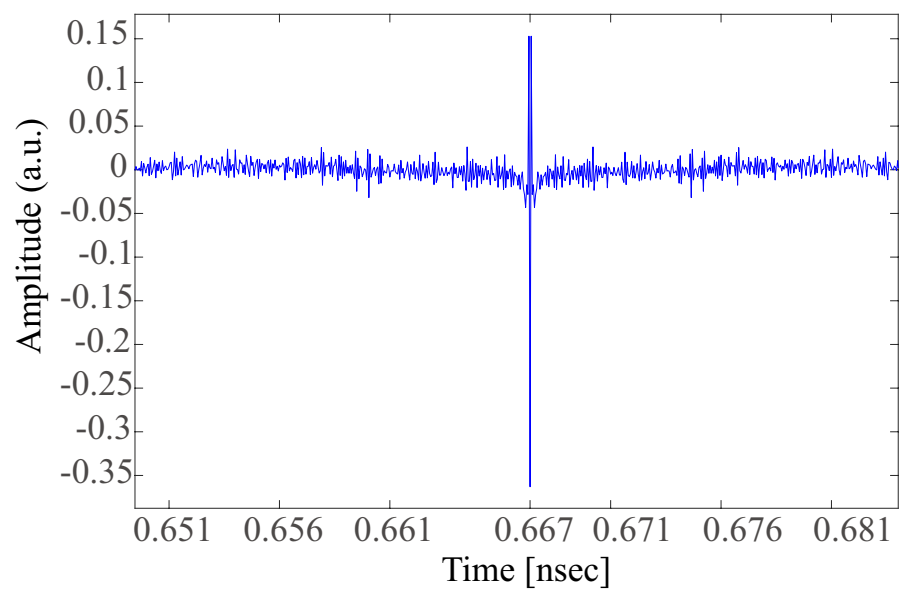

Fig. 4. Linear impulse response from wood. Incident angle $\theta=60 \mathrm{deg}$, distance $z=20 \mathrm{~cm}$, pressure $p=1007.9 \mathrm{hPa}, \mathrm{RH}=22.3 \%$, and temperature $T=295 \mathrm{~K}$.

where $\mathcal{F}^{-1}[\cdot]$ denotes the IFT operator.

If the channel has perfectly flat power spectrum with the linear phase, signal only experiences a time shift and it is perfectly causal after the time shift. In the general case, however, this is not always true. If the real and imaginary parts of the function are independent, such as in the case of real valued functions (zero phase), or linear phase imposed on real valued function, the resultant inverse Fourier transform is not causal (except for the special case of constant functions as discussed above). In this paper we are concerned with $\mathrm{THz}$ frequencies $(0.1-10 \mathrm{THz})$. In this case, the received signals will experience fading due to molecular absorption and the power spectrum is therefore never flat (unless we look at some narrow band within this range). Therefore, the impulse response based on the linear phase can not fulfill the causality if the channel experiences the frequency selectivity due to the molecular absorption.

For instance, we show the power spectrum of reflected path which is included the Rayleigh roughness factor in Fig. 3 for incident angle $\theta=60 \mathrm{deg}$, distance $z=20 \mathrm{~cm}$, pressure $p=1007.9 \mathrm{hPa}, \mathrm{RH}=22.3 \%$, and temperature $T=295$ $\mathrm{K}$. We also show the corresponding impulse response based on the linear phase shift calculated with (9)-(10) in Fig. 4. We can see that the main problem with the linear phase is that it causes a symmetric response around the zero (which is the propagation delay in the case of linear phase, and $\tau=$ $0.667 \mathrm{~ns}$ in this example). This effectively causes a response at the receiver before the arrival of the main peak. Therefore, utilization of the causal phase component should be considered in the calculation of the impulse response to ensure that the response is correct at the receiver.

\section{B. Minimum phase}

Impulse response with causality of the reflected path satisfies

$$
h_{\mathrm{C}, \text { refl }}(\tau)= \begin{cases}h_{\mathrm{C}, \mathrm{refl}}(\tau) & \tau \geq \tau_{m} \\ 0 & \tau<\tau_{m},\end{cases}
$$

where $\tau_{m}$ is the propagation delay of the reflected path and given by $\tau_{m}=z / c$. The frequency response of $h_{\mathrm{C}, \mathrm{refl}}(\tau)$ is given by

$$
\begin{aligned}
& H_{\text {refl }}(f, z)=\int_{\tau_{m}}^{\infty} h_{\mathrm{C}, \mathrm{refl}}(\tau, z) e^{-j 2 \pi f \tau} d \tau \\
& =e^{-j 2 \pi f \tau_{m}} \int_{0}^{\infty} h_{\mathrm{C}, \mathrm{refl}}\left(\tau+\tau_{m}, z\right) e^{-j 2 \pi f \tau} d \tau .
\end{aligned}
$$

$H_{\text {refl }}^{\prime}(f, z)$ is defined as causal frequency response in this paper and is given by

$$
H_{\text {refl }}^{\prime}(f, z)=\int_{0}^{\infty} h_{\mathrm{C}, \mathrm{refl}}\left(\tau+\tau_{m}, z\right) e^{-j 2 \pi f \tau} d \tau,
$$

which leads to

$$
H_{\text {refl }}(f, z)=e^{-j 2 \pi f \tau_{m}} H_{\text {refl }}^{\prime}(f, z) .
$$

Let $\exp [-\alpha(f, z)]$ and $\phi_{\mathrm{C}, \text { refl }}(f, z)$ denote the amplitude and phase components of $H_{\text {refl }}^{\prime}(f, z)$, respectively, i.e., $\exp [-\alpha(f, z)]=\left|H_{\text {refl }}^{\prime}(f, z)\right|=\left|H_{\text {refl }}(f, z)\right|$ and $\phi_{\mathrm{C}, \text { refl }}(f, z)=\arg \left(H_{\text {refl }}^{\prime}(f, z)\right)$. Then, $H_{\text {refl }}^{\prime}(f, z)$ is given by [15], [11]

$$
H_{\text {refl }}^{\prime}(f, z)=\exp \left[-\alpha(f, z)+j \phi_{\mathrm{C}, \mathrm{refl}}(f, z)\right] .
$$

$\alpha(f, z)$ and $\phi_{\mathrm{C}, \text { refl }}(f, z)$ are Hilbert transform pairs from the Kramers-Kronig relation [15], [11]. Therefore, the impulse response $h_{\mathrm{C}, \mathrm{refl}}(\tau, z)$ satisfies causality. Hence, $\phi_{\mathrm{C}, \mathrm{refl}}(f, z)$ is given by

$$
\phi_{\mathrm{C}, \mathrm{refl}}(f, z)=\frac{1}{\pi} \mathrm{PV} \int_{-\infty}^{\infty} \frac{\alpha\left(f^{\prime}, z\right)}{f-f^{\prime}} d f^{\prime},
$$

where PV represents Cauchy principal value [16]. The criterion to set the phase component in (16) is defined as minimum phase in this paper. Given $\left|H_{\text {refl }}(f, z)\right|, H_{\text {refl }}(f, z)$ is available based on (14)-(16). Finally, the impulse response with the minimum phase of the multipath is given by

$$
h_{\mathrm{C}, \text { refl }}(\tau)=\mathcal{F}^{-1}\left[H_{\text {refl }}(f, z)\right] \text {. }
$$




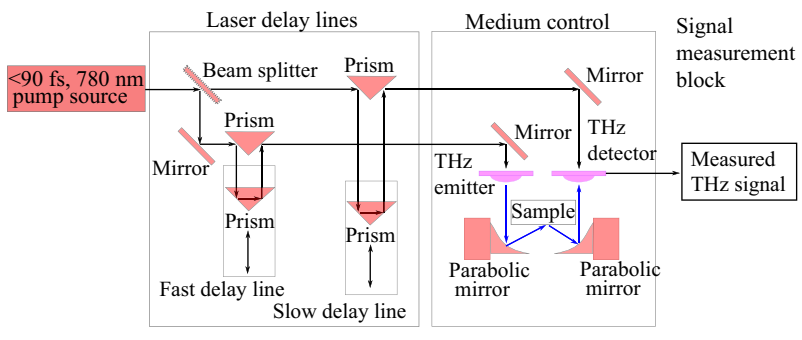

Fig. 5. Terahertz time domain spectroscopy setup.

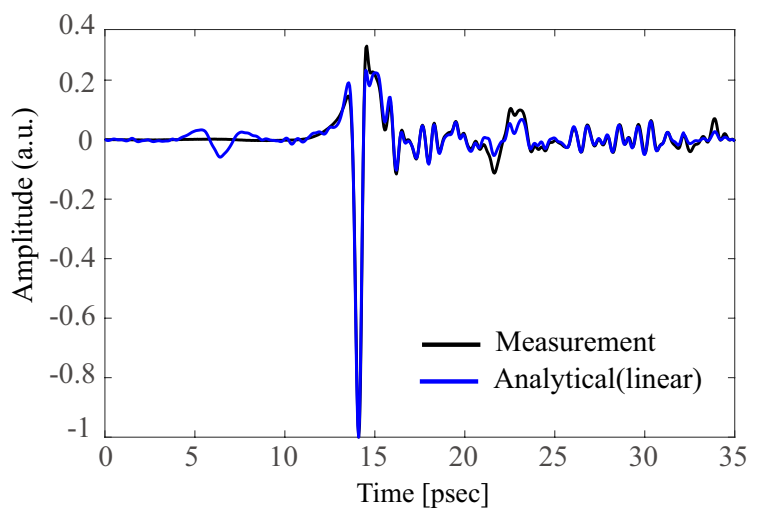

Fig. 6. Received pulse comparison between the measurement result at $\mathrm{RH}=$ $22.3 \%$ and incident angle $\theta=60 \mathrm{deg}$ and analytically obtained result based on the linear phase in the time-domain. Black line is received pulse based on measurement and blue line is received pulse based on linear phase.

From the above relation, the impulse response with minimum phase is calculated as follows. First, $\alpha(f, z)$ is obtained from (3) and (5) and then substituted in (16). Finally, (17) is obtained from (14), calculated using (15).

\section{NUMERICAL AND EXPERIMENTAL RESULTS}

In this section, we will show the validity of the impulse response with minimum phase. To valid impulse response of reflected path, we compare between the received pulse based on measurement and analysis. The measurement were made at distance $z=20 \mathrm{~cm}$, pressure $p=1007.9 \mathrm{hPa}, \mathrm{RH}=22.3 \%$, temperature $T=295 \mathrm{~K}$, and incident angle $\theta=60$. Measurements were conducted by terahertz time domain spectroscopy (THz-TDS) technique using the TeraView TeraPulse 4000. The experimental measurement setup is shown in Fig. 5. The measurement setup consists of the pump source, the laser delay lines, the medium control, and the signal measurement block. The delay lines are for obtaining $\mathrm{THz}$ pulse shape in time domain. The medium control is for setting channel medium. In order to measure $\mathrm{THz}$ signal in a reflected path, there is reflector at the sample location. In this results, wood is in the sample location. The emitter focus on the detector in $\mathrm{THz}$ optics in Fig. 5 with a beam width of approximately $2 \mathrm{~cm}$ at the sample location. The analytically obtained received pulse is obtained by the transmitted signal and the theoretical impulse responses $\left(h_{\mathrm{C}, \mathrm{refl}}, h_{\mathrm{L}, \mathrm{refl}}\right)$. The comparison received pulses are shown in Figs. 6 and 7, where $\mathrm{RH}=22.3 \%$ is assumed. The analytically obtained pulse from $h_{\mathrm{L}, \text { refl }}$ have

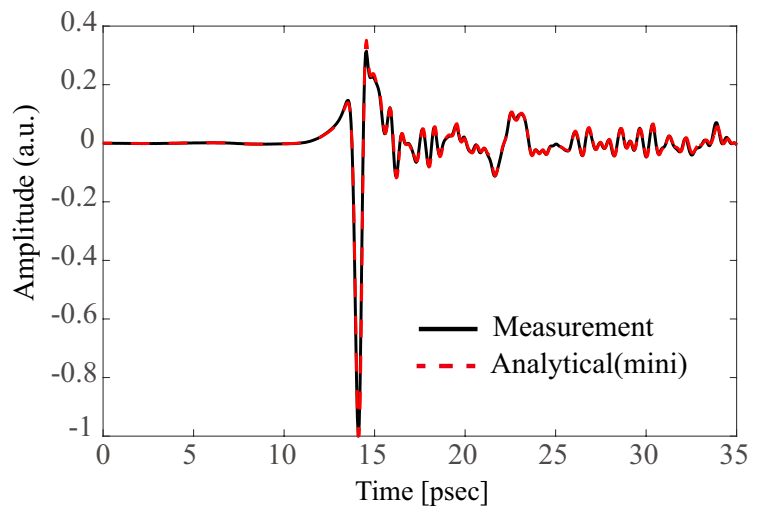

Fig. 7. Received pulse comparison between the measurement result at $\mathrm{RH}=$ $22.3 \%$ and incident angle $\theta=60 \mathrm{deg}$ and analytically obtained result based on the minimum phase in the time-domain. Black line is received pulse based on measurement and red line is received pulse based on minimum phase.

the signal at around $5 \mathrm{psec}$ and at around $22 \mathrm{psec}$ the signal value is smaller than measurement signal. On the other hand, the analytically obtained pulse from $h_{\mathrm{C} \text {,refl }}$ agrees with the received pulse from measurement data very well.

\section{CONCLUSION}

This paper is introduced impulse response of the reflected pulse from rough surface for $\mathrm{THz}$ band. For causality, we obtain minimum phase from power spectrum of reflected path and apply minimum phase to frequency response of reflected path. Comparison of the measurement data is valid our proposed method.

\section{ACKNOWLEDGMENT}

The work of J. Kokkoniemi and J. Lehtomaki in TERRANOVA project has received funding from Horizon 2020, European Union's Framework Programme for Research and Innovation, under grant agreement No. 761794

\section{REFERENCES}

[1] S. Cherry, "Edholm's law of bandwidth," IEEE Spectrum, vol. 41, no. 7, pp. 58-60, Jul. 2004.

[2] R. Piesiewicz, C. Jansen, D. Mittleman, T. Kleine-Ostmann, M. Koch, and T. Kurner, "Scattering analysis for the modeling of thz communication systems," IEEE Transactions on Antennas and Propagation, vol. 55, no. 11, pp. 3002-3009, Nov. 2007.

[3] B. Sklar, "Rayleigh fading channels in mobile digital communication systems part I: Characterization," IEEE Communications Magazine, vol. 35, no. 7, pp. 90-100, Jul 1997.

[4] J. M. Jornet and I. F. Akyildiz, "Channel modeling and capacity analysis for electromagnetic wireless nanonetworks in the terahertz band," IEEE Transactions on Wireless Communications, vol. 10, no. 10, pp. 32113221 , Oct 2011.

[5] J. Kokkoniemi, J. Lehtomäki, K. Umebayashi, and M. Juntti, "Frequency and time domain channel models for nanonetworks in terahertz band," IEEE Transactions on Antennas and Propagation, vol. 63, no. 2, pp. 678-691, Feb 2015.

[6] J. M. Jornet and I. F. Akyildiz, "Femtosecond-long pulse-based modulation for terahertz band communication in nanonetworks," IEEE Transactions on Communications, vol. 62, no. 5, pp. 1742-1754, May 2014.

[7] C. Han, A. O. Bicen, and I. F. Akyildiz, "Multi ray channel modeling and wideband characterization for wireless communications in the terahertz band," IEEE Transactions on Wireless Communications, vol. 14, no. 5, pp. 2402-2412, May 2015. 
[8] K. Tsujimura, K. Umebayashi, J. Kokkoniemi, J. Lehtomaki, and Y. Suzuki, "A causal channel model for the terahertz band," IEEE Transactions on Terahertz Science and Technology, vol. 8, no. 1, pp. 52-62, Jan 2018.

[9] J. Kokkoniemi, V. Petrov, D. Moltchanov, J. Lehtomäki, Y. Koucheryavy, and M. Juntti, "Wideband terahertz band reflection and diffuse scattering measurements for beyond 5g indoor wireless networks," in European Wireless 2016; 22th European Wireless Conference; Proceedings of, 2016, pp. 1-6.

[10] S. Priebe, M. Jacob, C. Jansen, and T. Kürner, "Non-specular scattering modeling for thz propagation simulations," in Antennas and Propagation (EUCAP), Proceedings of the 5th European Conference on. IEEE, 2011, pp. $1-5$.

[11] S. Paine, "The am atmospheric model," Smithsonian Astrophysical Observatory, SMA Technical Memo 152, Feb 2012.

[12] L. S. Rothman et al., "The HITRAN 2008 molecular spectroscopic database," Journal of Quantitative Spectroscopy and Radiative Transfer, vol. 110, no. 9-10, pp. 533-572, Jun-July 2009.

[13] S. J. Adams, Electromagnetic theory. McGraw-Hill BookCompany, 1941.

[14] C. Jansen et al., "Diffuse scattering from rough surfaces in thz communication channels," IEEE Transactions on Terahertz Science and Technology, vol. 1, no. 2, pp. 462-472, Nov. 2011.

[15] H. Kuzmany, Solid-State Spectroscopy. Springer, 1998.

[16] J. Alan and Z. Daniel, Table of Integrals, Series, and Products, 7th ed. Academic Press, 2007. 